\title{
OHNE FLEISS KEIN REIS
}

\section{Liebe Kolleginnen und Kollegen!}

„Bila wali siwesi kushiba“. Das ist für einmal nicht Chinesisch, sondern Swahili, ein Satz, den wir von unseren Angestellten in Tansania in Ostafrika immer wieder gehört haben: „Ohne Reis kann ich nicht satt werden.“ Dies gilt auch für die Hälfte der Weltbevölkerung, für welche Reis das wichtigste Grundnahrungsmittel darstellt. Reis gehört zur Familie der Süßgräser, Gattung Oryza, wovon es zwei domestizierte Sorten gibt: Oryza sativa und Oryza glaberrima. Unter dem Namen „Wildreis“, auch Indianerreis oder kanadischer Reis genannt, wird eine andere Pflanzengattung, nämlich der Wasserreis (Zizania) verstanden, der keine wilde Form des Reises (Oryza) darstellt. Seine 1-2 cm langen grünen Früchte (Körner) werden bei der Trocknung dunkelbraun bis schwarz.

In vielen Sprachen gibt es - je nach Verarbeitungsstufe - verschiedene Bezeichnungen für den Reis: Auf Swahili heißt der Reis auf dem Feld mpunga, der geerntete, enthülste Reis mchele, gestampfter Reis wird mit pepeta und gekochter Reis mit wali bezeichnet.

Im Chinesischen wird die Reispflanze dào genannt, die Reiskörner dàmi und der gekochte Reis mifàn. Die Bedeutung von Reis in der chinesischen Ernährung zeigt auch die Redewendung ,ni chi fan le ma?“ (Hast Du schon Reis gegessen?), eine nebst „Ni hao“ häufige Begrüßungsformel. Auch in Afrika (Tansania) ist eigentlich nur Reis eine richtige Mahlzeit. Deshalb muss der Chirurg, wenn er wissen will, ob der Patient nüchtern ist, eben nicht nur fragen: „Hast Du etwas gegessen?“, sondern: „Hast Du Reis gegessen?"“ Sonst hat er auf einmal einen Patienten mit vollem Magen auf dem Operationstisch.

Im Westen gilt Reis als Bestandteil der chinesischen Küche par excellence. Dabei wird Reis hauptsächlich im Süden Chinas in der Kantonesischen Küche serviert, während im Norden Speisen auf (Weizen-)Mehl-Basis wie Nudeln oder Teigtaschen dominieren. Der Yangzi-Fluss bildet sozusagen die Nudel/Reis-Grenze. Oft wird Reis erst am Ende einer Mahlzeit als Sättigungsbeilage aufgetischt.

Die Domestikation der Reispflanze hat, wie diverse Studien nachweisen konnten, vor mehr als 8000 Jahren in der Provinz Guangdong im Delta des Perlflusses (zwischen Macau und Hongkong) stattgefunden und sich von dort über ganz Südostasien bis Indien verbreitet. Es waren die Mauren, die im 10. Jahrhundert den Reis in Spanien ein- geführt haben. Die arabische Bezeichnung ,al ruzz“ hat sich im spanischen und portugiesischen Wort für Reis erhalten:Arroz. Die nördlichste Reisanbaufläche der Welt liegt übrigens im Maggia-Delta und in der MagadinoEbene in der Schweiz im Kanton Tessin, wo jährlich 400 Tonnen Reis geerntet werden. In derVolksrepublik China werden jährlich 212 Millionen Tonnen Reis produziert, die weltweite Produktion liegt bei 782 Millionen Tonnen. Der Reisanbau ist nach Schätzungen des Weltklimarates (IPCC) einer der Hauptgründe für steigende Emissionen von Methan. Kohlendioxid macht $70 \%$ der Treibhausgase aus, Methan 23\%, es wirkt aber 25-mal stärker als $\mathrm{CO}_{2}$. Und dieses steigt nicht nur aus den sumpfigen Reisfeldern, sondern wird auch von Kühen verursacht. Eine Kuh belastet das Klima so stark wie ein Auto, bei 1,5 Milliarden Kühen kommt doch einiges zusammen. In den USA fragte der Kongressabgeordnete Jim Sensenbrenner, ob man den Kühen jetzt Katalysatoren in den Hintern stopfen müsse. Doch da liegt Jim 180 Grad verkehrt: Das Methan bei Kühen entweicht nicht etwa hinten, sondern wird gerülpst. Das Methan entsteht durch den mikrobiellen Futterabbau und wird beim Wiederkäuen mit dem vorverdauten Futter aus dem Pansen (Vormagen) hochgewürgt. Es gibt mehr als 8000 Sorten Oryza sativa: Für weißen Reis wird Vollkornreis gemahlen, wobei Schale, Kleie und Keimlinge entfernt werden, ist also polierter Reis. Er ist so länger haltbar, da die Fettstoffe entfernt werden. Ebenso entfernt wird aber auch das Vitamin B1, das Thiamin (auch Aneurin genannt).Vitamin-B1-Mangel ist eine klassische Avitaminose, die unter dem Namen Beriberi bekannt wurde (Singhalesisch für: „Ich kann nicht“). Sie tritt auf bei vorwiegender Ernährung mit geschältem Reis, aber auch bei Alkoholikern und ist gekennzeichnet durch Muskelschwächen, Ataxie, Sprachstörungen, Kopfschmerzen, Herzinsuffizienz und Ödemen. Unter der Bezeichnung Jiao Qi war eine Krankheit, deren Symptome mit Beriberi übereinstimmen, bereits im antiken China bekannt. Im Chinese Medical Journal zitieren Hou und Yu die Erwähnung von Beriberi im Jahr 344 BC. Beim roten Reis gilt es zu unterscheiden zwischen dem roten Camargue-Reis, der seine tief granatrote Farbe von den tonhaltigen Böden Südfrankreichs bekommt. Außerdem gibt es den rot fermentierten Reis, der aus China stammt und seine Farbe diversen Rotschimmel-Pilzen verdankt, welche die Fermentation anregen. Dadurch entsteht der 
Stoff Monakolin K, der chemisch identisch ist mit dem Cholesterinsenker Lovastatin und auch ähnliche Nebenwirkungen zeigt, nämlich Myopathien und Leberschäden. Schwarzer Reis ist eine echte Oryza-Sorte und nicht zu verwechseln mit dem indianischen Wasserreis, der zur Gattung Zizania (Wassergräser) gehört. Der echte schwarze Reis ist ein Vollkornreis, der nicht geschält wird und so seine wertvollen Inhaltstoffe behalten kann. Seine dunkle Farbe stammt von den Anthocyanen, den wertvollen Antioxidanzien. Der Genuss des schwarzen Reises war ursprünglich ausschließlich dem chinesischen Kaiser vorbehalten. Deshalb wurde er auch „,verbotener Reis“ genannt. Der grüne Reis heißt auch Aplatireis und stammt hauptsächlich aus Vietnam und Thailand. Er ist keine spezielle Reissorte, sondern wird noch unreif geerntet. Es gibt Nassreis-Anbau und Trockenreis-Anbau. Um 1 kg Nassreis zu gewinnen, benötigt man zwischen 3000 und $5000 \mathrm{Li}-$ ter fließendes Wasser. Mit den typischen, terrassenförmig angelegten Reisfeldern können Hanglagen bis $2000 \mathrm{~m}$ bewirtschaftet werden. Durch ein ausgeklügeltes Netz von Bewässerungskanälen wird das Wasser von der obersten bis zur untersten Terrasse verteilt. Diese Kanäle werden in der TCM mit den Energieleitbahnen, den Meridianen, verglichen und das darin fließende Wasser mit dem Energiefluss des Qi. Die Felder werden mit einem Pflug vorbereitet, der von Menschen (Abb. 1) oder einem Wasserbüffel gezogen wird, dann werden die in Saatbeeten vorgezogenen Jungpflanzen im Abstand von $20 \mathrm{~cm}$ in die gefluteten Felder gesetzt. Das typische Bild der gebückt arbeitenden Reisbauern dient auch als Metapher von Yin und Yang: In dieser Haltung brennt die Sonne auf den Rücken, daher befindet sich Yang auf der Rückseite des Menschen und Yin vorne im Schatten.

Nach 5-6 Monaten ist der Reis erntereif. Die goldgelben Rispen werden mit Sicheln geschnitten und an der Sonne getrocknet. Nach dem Dreschen werden die Reiskörner ,geworfelt“, d.h. mit flachen Körben in die Luft geworfen, wodurch die Spreu vom Reis getrennt wird. Nicht mit Sicheln, sondern mit Schneckenhäusern der Afrikanischen Riesenschnecke (Achatina) wird der trocken angebaute Reis in Ostafrika geerntet. Eine relativ mühsame Angelegenheit, wie wir selbst erfahren durften. Mit einer Sense, die wir aus der Schweiz kommen ließen, hat

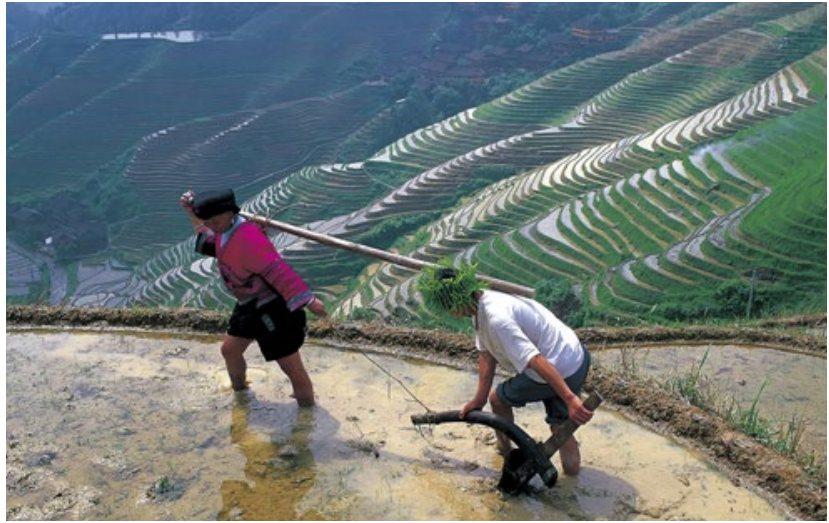

Abb. 1: Reisterrassen von Longsheng, Angehörige der Yao-Minderheit beim Pflügen, (C) chinatours.de

unser Gärtner dann sein Reisfeld in einem Bruchteil der „normalen“ Zeit abgeerntet, was von zahlreichen $\mathrm{Zu}$ schauern als Sensation begutachtet wurde. So geht nachhaltige Entwicklungshilfe:Vom Schneckenhaus zur Sense - und nicht zum Traktor!

Reis gilt als Symbol für Glück, Fruchtbarkeit und ein langes Leben. Darauf deutet der weitverbreitete Brauch, das Brautpaar mit Reis zu bewerfen und ihm dadurch Glück zu bringen. Schon Hippokrates sagte bekanntlich: „Lasset Nahrung unsere Medizin sein.“ Aber nirgends wird der fließende Übergang zwischen Nahrungsmittel und Heilmittel deutlicher als in der TCM. Im Standardwerk „Chinese Medical Herbology and Pharmacology“ von John und Tina Chen ist der Reis (chinesisch: Geng Mi oder Da Mi, Semen oryzae) bei den tonisierenden Mitteln aufgeführt: Er stärkt das Lungen-Qi und den mittleren Dreierwärmer. Da der Reis dem Element Erde zugeordnet wird, unterstützt er auch den Funktionskreis Milz und Magen. Lakonisch heißt es am Schluss des kurzen Abschnitts: „This herb is commonly used as food“. Diese Feststellung ist für Alle, die diesen Artikel gelesen haben, nicht wirklich eine Überraschung.

Ihr

A.Wirz-Ridolfi

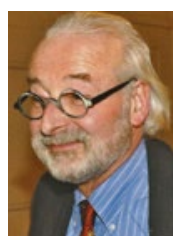

Dr. med. Andreas Wirz-Ridolfi

Chirurgie FMH, Akupunktur/TCM

Medi-China $A G$,

Im langen Loh 11, 4054 Basel/Schweiz

Tel.: +41613016585 